\title{
Prévalence du trouble du spectre de l'alcoolisation fotale (TSAF) chez les enfants et les adolescents : résultats de l'Enquête canadienne sur la santé des enfants et des jeunes de 2019
}

\author{
Sarah Palmeter, MSP; Adam Probert, M. Sc.; Claudia Lagacé, M. Sc.
}

Diffuser cet article sur Twitter

\section{Résumé}

Introduction. L'absence d'estimations de la prévalence nationale du trouble du spectre de l'alcoolisation fœtale (TSAF) constitue une lacune importante dans les connaissances.

\begin{abstract}
Méthodologie. La prévalence du TSAF, déterminée à l'aide des données de l'Enquête canadienne sur la santé des enfants et des jeunes de 2019, a été analysée en fonction de l'âge, du sexe et de l'identité (autochtone ou non autochtone). L'âge médian au moment du diagnostic et les problèmes de santé concomitants de longue durée ont aussi été examinés.
\end{abstract}

Résultats. La prévalence du TSAF chez les enfants et les adolescents canadiens vivant en ménage privé était de 1 pour 1000 (0,1\%). Cette prévalence était considérablement plus élevée chez ceux s'étant identifiés comme Autochtones et vivant hors réserve $(1,2 \%)$.

Conclusion. Ces observations corroborent les résultats des études sur la prévalence du TSAF ayant utilisé des méthodes de surveillance passive. Elles constituent un point de départ vers une meilleure compréhension de la prévalence et du fardeau du TSAF au Canada.

Mots-clés : trouble du spectre de l'alcoolisation fœtale, prévalence, surveillance de la santé publique, santé des enfants, Enquête canadienne sur la santé des enfants et des jeunes

\section{Introduction}

L'expression « trouble du spectre de l'alcoolisation fœtale ", ou TSAF, est un terme diagnostique utilisé pour décrire les conséquences de l'exposition prénatale à l'alcool sur le cerveau et l'organisme ${ }^{1,2}$. Le TSAF dure toute la vie. Les personnes atteintes du TSAF éprouveront des difficultés dans leur vie quotidienne et auront besoin, pour réaliser leur potentiel, d'aide à la motricité, à la santé physique, à l'apprentissage, à la mémoire, à l'attention, à la communication, à la régulation des émotions et aux compétences sociales.
Chaque personne atteinte du TSAF est unique et a des forces et des faiblesses qui lui sont propres ${ }^{3}$.

$\mathrm{Au}$ cours des dernières décennies, une quantité importante de données probantes a été publiée en ce qui a trait à la prévention, à l'identification et au diagnostic précoce du TSAF ainsi que sur l'estimation de sa prévalence ${ }^{4}$. Parmi les méthodes utilisées et testées pour estimer la prévalence, la plupart se sont intéressées à des zones géographiques particulières et à des populations vulnérables ${ }^{5-7}$. Les estimations de prévalence au Canada ne sont pas uniformes
Points saillants

- En 2019, la prévalence du TSAF chez les enfants et les adolescents canadiens âgés de 1 à 17 ans vivant en ménage privé était de 1 pour $1000(0,1 \%)$.

- La prévalence du TSAF était nettement plus élevée chez les enfants et les adolescents canadiens s'étant identifiés comme Autochtones et vivant hors réserve que chez ceux ne s'étant pas identifiés comme Autochtones (1,2\% contre 0,1\%).

- Lâge médian au moment du diagnostic de TSAF chez les enfants et les adolescents était de 5,7 ans.

- La plupart des enfants et des adolescents atteints du TSAF avaient au moins un autre problème de santé de longue durée.

- Une surveillance du TSAF à l'échelle nationale est nécessaire pour éclaircir la prévalence de ce problème de santé.

ni d'envergure nationale. La prévalence du TSAF est, du fait de la nature de ce trouble, difficile à mesurer, principalement en raison de la complexité de sa reconnaissance, de son dépistage et de son diagnostic $^{8}$. Il n'existe aucun marqueur biologique ou génétique propre au TSAF. Par ailleurs, un grand nombre d'enfants affectés par l'exposition prénatale à l'alcool ne présentent pas les traits physiques associés au TSAF que sont par exemple les dysmorphies faciales ${ }^{9}$. Les lignes directrices canadiennes en matière

Rattachement des auteurs :

Agence de la santé publique du Canada, Ottawa (Ontario), Canada

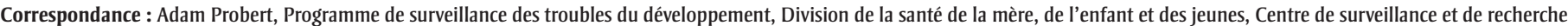

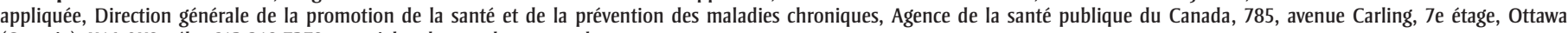
(Ontario) K1A 0K9; tél. : 613-218-7379; courriel : adam.probert@canada.ca 
de diagnostic exigent une information détaillée - qui n'est pas toujours disponible - sur l'exposition prénatale à l'alcool afin de pouvoir avoir confirmation du diagnostic $^{9}$. Il est donc difficile d'établir la présence du TSAF dans les cas qui ne sont pas sévères ${ }^{1}$.

L'absence d'estimations de la prévalence nationale du TSAF constitue une lacune importante dans les connaissances utiles aux interventions en santé publique. Cet article vise à fournir les premières estimations de la prévalence nationale du TSAF, fondées sur les données l'Enquête canadienne sur la santé des enfants et des jeunes (ECSEJ) de 2019.

\section{Méthodologie}

L'ECSEJ de 2019 a porté sur un échantillon national de Canadiens de 1 à 17 ans (en date du 31 janvier 2019) vivant en ménage privé dans les dix provinces et les trois territoires du pays $(n=39951$; taux de réponse $=52,1 \%)^{10,11}$. Les enfants et les adolescents vivant dans des réserves des Premières Nations et d'autres établissements autochtones des provinces, dans des foyers d'accueil et en institution n'étaient pas éligibles à l'enquête ${ }^{10}$. Les données ont été collectées au moyen d'un questionnaire à remplir par la personne la mieux renseignée sur l'enfant ou l'adolescent (1 à 17 ans) ${ }^{11}$.

Les enfants et les adolescents ont été considérés comme atteints du TSAF si leur répondant a répondu " oui » pour " L'ensemble des troubles causés par l'alcoolisation fœtale, aussi appelé ETCAF » à la question « [L'enfant] a-t-il été diagnostiqué avec l'un des problèmes de santé de longue durée suivants? $»^{12}$.

Les caractéristiques sociodémographiques et sanitaires incluses dans cette étude sont l'âge (1 à 11 ans ou 12 à 17 ans), le sexe (masculin ou féminin) et l'identité autochtone ou non autochtone (membre des Premières Nations, des Métis ou des Inuits ou non-Autochtone).

Lâge médian au moment du diagnostic a été établi à l'aide de la question : "Quel âge avait-il quand il a été diagnostiqué pour la première fois avec les problèmes de santé suivants? - L'ensemble des troubles causés par l'alcoolisation fœtale, aussi appelé ETCAF $»^{12}$.

Les enfants et les adolescents ont été considérés comme atteints d'un problème de santé de longue durée si leur répondant a répondu « oui » à la question « [L'enfant] a-t-il été diagnostiqué avec un ou l'autre des problèmes de santé de longue durée suivants? » pour l'un des problèmes de santé parmi asthme, diabète, épilepsie, trouble d'anxiété, trouble de l'humeur, trouble de l'alimentation, difficulté d'apprentissage ou trouble d'apprentissage, trouble du déficit de l'attention ou trouble du déficit de l'attention avec hyperactivité (aussi appelé TDA/TDAH), trouble du spectre de l'autisme et tout autre problème de santé de longue durée ${ }^{12}$.

Des analyses descriptives ont été réalisées pour établir la prévalence du TSAF chez les enfants et les adolescents canadiens. Les estimations concernant les enfants et les adolescents atteints du TSAF ont été comparées avec celles concernant les enfants et les adolescents n'ayant pas eu ce diagnostic. Toutes les estimations ont été pondérées de manière à être représentatives de la population canadienne âgée de 1 à 17 ans. Nous avons utilisé la méthode de ré-échantillonnage " bootstrap » pour établir des estimations de la variance, avec des intervalles de confiance (IC) à $95 \%$ et des coefficients de variation. Toutes les analyses ont été réalisées à l'aide du logiciel statistique SAS Enterprise Guide (version 7.1) ${ }^{13}$. Les résultats ont été supprimés dans les cas où la variabilité d'échantillonnage était élevée (coefficient de variation supérieur à $35 \%$ ).

\section{Résultats}

En 2019, environ 9400 enfants et adolescents canadiens de 1 à 17 ans (selon un échantillon non pondéré de 54 sujets), soit une proportion de 1 pour 1000 , avaient reçu un diagnostic de $\operatorname{TSAF}(0,1 \%$; ; IC à $95 \%$ : 0,1 à 0,2) (tableau 1). Cela correspond à 1 pour 1000 enfants de 1 à 11 ans $\left(0,1 \%{ }^{\mathrm{D}}\right.$; IC à $95 \%: 0,0$ à 0,2$)$ et 2 pour 1000 adolescents de 12 à 17 ans $(0,2 \%$; IC à $95 \%: 0,1$ à 0,3). La prévalence était la même chez les filles et chez les garçons.

Bien que nous disposions de données permettant d'analyser diverses caractéristiques socioéconomiques comme l'origine ethnique (appartenance ou non à une minorité visible), le milieu de vie (urbain ou

TABLEAU 1

Prévalence du trouble du spectre de l'alcoolisation foetale (TSAF) chez les enfants et les adolescents âgés de 1 à 17 ans, selon certaines caractéristiques individuelles, Canada, 2019

\begin{tabular}{|c|c|}
\hline Caractéristiques individuelles & $\begin{array}{l}\text { Prévalence du TSAF en pourcentage (\%) } \\
\text { avec IC à } 95 \% \\
(n=9382)\end{array}$ \\
\hline \multicolumn{2}{|l|}{ Âge (ans) } \\
\hline 1 à 11 & $0,1(0,0 \text { à } 0,2)^{\mathrm{D}}$ \\
\hline 12 à 17 & $0,2(0,1 \text { à } 0,3)^{\mathrm{C}}$ \\
\hline \multicolumn{2}{|l|}{ Sexe } \\
\hline Masculin & $0,1(0,1 \text { à } 0,2)^{\mathrm{D}}$ \\
\hline Féminin & $0,1(0,1 \text { à } 0,2)^{\mathrm{D}}$ \\
\hline \multicolumn{2}{|l|}{ Identité autochtone } \\
\hline Autochtone (Premières Nations, Métis, Inuit) & $1,2(0,4 \text { à } 1,9)^{\mathrm{D}}$ \\
\hline Non autochtone & $0,1(0,1 \text { à } 0,1)^{\mathrm{C}}$ \\
\hline Total & $0,1(0,1 \text { à } 0,2)^{\mathrm{C}}$ \\
\hline
\end{tabular}

Source : Enquête canadienne sur la santé des enfants et des jeunes de 2019.

Abréviations : IC, intervalle de confiance; TSAF, trouble du spectre de l'alcoolisation fotale.

${ }^{c}$ Variabilité d'échantillonnage élevée (coefficient de variation entre 15,0\% et 25,0\%).

${ }^{D}$ Variabilité d'échantillonnage élevée (coefficient de variation entre 25,0 \% et 35,0\%).

Remarques : Les pourcentages et les intervalles de confiance à $95 \%$ sont fondés sur des données pondérées. Les estimations pondérées sont fondées sur un échantillon non pondéré de 54 sujets.

C Variabilité d'échantillonnage élevée (coefficient de variation entre 15,0 \% et 25,0\%).

${ }^{D}$ Variabilité d'échantillonnage élevée (coefficient de variation entre 25,0 \% et 35,0\%). 
rural), le revenu et le niveau de scolarité, les estimations fondées sur ces données présentaient une trop grande variabilité d'échantillonnage pour pouvoir être présentées.

La prévalence du TSAF chez les enfants et adolescents autochtones vivant hors réserve était de $1,2 \%^{\mathrm{D}}$ (IC à $95 \%: 0,4$ à 1,9 ), contre $0,1 \%{ }^{\mathrm{C}}$ (IC à $95 \%: 0,1$ à 0,1 ) chez les enfants non autochtones (tableau 1). Cette différence est statistiquement significative, mais elle doit être interprétée avec prudence en raison des coefficients de variation élevés de ces deux estimations.

Lâge médian des enfants et des adolescents au moment du diagnostic de TSAF était de 5,7 ans (IC à $95 \%: 3,8$ à 7,6) et aucune différence statistiquement significative n'est ressortie lorsque les données ont été analysées en fonction du sexe ou d'autres caractéristiques sociodémographiques (données non présentées).

Le TSAF est associé à un grand nombre de problèmes de santé concomitants ${ }^{14}$. La majorité $(82,7 \%$; IC à $95 \%: 66,5$ à 98,9$)$ des enfants et des adolescents atteints du TSAF avaient au moins un autre problème de santé de longue durée, contre un peu moins du quart $(23,4 \%$; IC à $95 \%: 22,8$ à 24,0) de ceux sans TSAF. Chez les enfants et adolescents de 5 à 17 ans, 64,7 \% ${ }^{\mathrm{C}}$ (IC à $95 \%: 45,1$ à 84,3) avaient reçu un diagnostic de trouble ou de difficulté d'apprentissage; $44,3 \%{ }^{\mathrm{C}}$ (IC à $95 \%$ : 25,3 à $63,3)$ avaient reçu un diagnostic de trouble d'anxiété et $32,9 \%$ D (IC à $95 \%: 14,7$ à 51,1) avaient reçu un diagnostic de trouble de l'humeur (tableau 2). Chez les enfants et adolescents de 1 à 17 ans, 69,6\% (IC à $95 \%$ : 51,5 à 87,7) avaient reçu un diagnostic de TDA/TDAH et $31,9 \%{ }^{\mathrm{D}}$ (IC à $95 \%: 13,6$ à 50,2) avaient reçu un diagnostic d'autres problèmes de santé de longue durée (tableau 2).

\section{Analyse}

D'après les données de l'ECSEJ de 2019, la prévalence nationale du TSAF diagnostiqué chez les enfants et adolescents âgés de 1 à 17 ans est de 1 pour 1000 , soit $0,1 \%{ }^{\mathrm{C}}$. Aucune différence statistiquement significative n'a été constatée entre les deux sexes, mais la prévalence du TSAF était plus élevée chez les enfants et les

TABLEAU 2

Problèmes de santé de longue durée chez les enfants et les adolescents âgés de 1 à 17 ans, avec trouble du spectre de l'alcoolisation fotale (TSAF) et sans TSAF, Canada, 2019

\begin{tabular}{|c|c|c|}
\hline $\begin{array}{l}\text { Problème de santé de longue } \\
\text { durée (plage d'âge en années) }\end{array}$ & $\begin{array}{l}\text { Avec TSAF, en \% (IC à } 95 \%) \\
(\mathbf{n}=9 \text { 382) }\end{array}$ & $\begin{array}{l}\text { Sans TSAF, en \% (IC à } 95 \%) \\
(n=6557361)\end{array}$ \\
\hline \multicolumn{3}{|c|}{ Difficulté ou trouble d'apprentissage (5 à 17) } \\
\hline Oui & $64,7(45,1 \text { à } 84,3)^{\mathrm{C}}$ & 8,4 (7,9 à 8,8) \\
\hline Non & $35,3(15,7 \text { à } 54,9)^{\mathrm{D}}$ & $91,6(91,2$ à 92,1$)$ \\
\hline \multicolumn{3}{|l|}{ Trouble d'anxiété (5 à 17) } \\
\hline Oui & $44,3(25,3 \text { à } 63,3)^{\mathrm{c}}$ & $5,1(4,8$ à 5,5$)$ \\
\hline Non & $55,7(36,7 \text { à } 74,7)^{\mathrm{C}}$ & $94,9(94,5$ à 95,2$)$ \\
\hline \multicolumn{3}{|l|}{ Trouble de l'humeur (5 à 17) } \\
\hline Oui & $32,9(14,7 \text { à } 51,1)^{\mathrm{D}}$ & $2,1(1,8$ à 2,3$)$ \\
\hline Non & $67,1(48,9$ à 85,3$)$ & $97,9(97,7$ à 98,2$)$ \\
\hline \multicolumn{3}{|l|}{ TDA/TDAH (1 à 17) } \\
\hline Oui & $69,6(51,5$ à 87,7$)$ & 6,7 (6,3 à 7,1) \\
\hline Non & $30,4(12,3 \text { à } 48,5)^{\mathrm{D}}$ & 93,3 (92,9 à 93,7) \\
\hline \multicolumn{3}{|c|}{ Autre problème de santé de longue durée (1 à 17) } \\
\hline Oui & $31,9(13,6 \text { à } 50,2)^{\mathrm{D}}$ & $6,7(6,4$ à 7,1$)$ \\
\hline Non & $68,1(49,8$ à 86,4) & $93,3(92,9$ à 93,6) \\
\hline
\end{tabular}

Source : Enquête canadienne sur la santé des enfants et des jeunes de 2019.

Abréviations : IC, intervalle de confiance; TDA/TDAH, trouble du déficit de l'attention ou trouble du déficit de l'attention avec hyperactivité; TSAF, trouble du spectre de l'alcoolisation foetale.

C Variabilité d'échantillonnage élevée (coefficient de variation entre 15,0\% et 25,0\%).

${ }^{\mathrm{D}}$ Variabilité d'échantillonnage élevée (coefficient de variation entre 25,0 \% et 35,0\%).

Remarques : Les pourcentages et les intervalles de confiance à $95 \%$ sont fondés sur des données pondérées. Les estimations pondérées sont fondées sur deux échantillons non pondérés, l'un de 54 sujets (enfants et adolescents atteints d'un TSAF) et l'autre de 39897 sujets (enfants et adolescents sans TSAF).

Le nombre d'enfants et adolescents atteints d'un TSAF ayant aussi de l'asthme, du diabète, de l'épilepsie, un trouble de l'alimentation ou un trouble du spectre de l'autisme était insuffisant pour présenter des estimations concernant ces problèmes de santé.

adolescents s’identifiant comme Autochtones et vivant hors réserve.

Les études sur la prévalence du TSAF au Canada reposent sur diverses méthodes, dont des examens cliniques et la détermination active des cas, tout en s'intéressant à des populations, à des zones géographiques et à des tranches d'âge différentes ${ }^{5,15}$. Les estimations canadiennes publiées vont de 0,09\%, avec un instrument de mesure du développement de la petite enfance $^{5}$, à entre $1,4 \%$ et $4,4 \%$, avec des méthodes de détermination active des cas $^{15,16}$. Des études semblables menées en Australie, aux États-Unis et en Europe de l'Ouest, mais avec des méthodes différentes et sur des tranches d'âge différentes, ont établi une prévalence du TSAF comprise entre $1 \%$ et $5 \%$ chez les enfants ch,17-20 $^{6}$. Notre résultat de $0,1 \%$, qui se situe à la limite inférieure de cette plage de valeurs, correspond aux valeurs reposant sur des études fondées sur la détermination passive des cas $^{5}$, mais il est nettement inférieur aux valeurs reposant sur des études fondées sur la détermination active des cas $^{15}$.

La prévalence du TSAF était significativement plus élevée chez les enfants et les adolescents canadiens d'identité autochtone et vivant hors réserve que chez les autres (1,2 \% contre 0,1\%). Les données de l'ECSEJ ne permettent pas d'analyser les différences sur le plan des méthodes de diagnostic, de l'accès aux soins ou d'autres facteurs susceptibles d'expliquer cet écart de prévalence. Toutefois, cette estimation correspond aux résultats d'autres études menées sur les populations autochtones ${ }^{21}$. Dans une de ces études, la prévalence globale du TSAF chez les enfants et adolescents autochtones vivant dans une réserve et hors réserve au Canada était de $8,7 \%$, comparativement à 0,5\% au sein

C Variabilité d'échantillonnage élevée (coefficient de variation entre 15,0 \% et 25,0\%).

${ }^{D}$ Variabilité d'échantillonnage élevée (coefficient de variation entre 25,0\% et 35,0\%). 
de la population générale ${ }^{21}$. Des tendances semblables ont été constatées chez les enfants et les adolescents en AustralieOccidentale et dans le sud-ouest des États-Unis, où vivent aussi d'importantes populations autochtones ${ }^{6,7,22}$. Ces études ont estimé la prévalence du TSAF entre $2 \%{ }^{6}$ et près de $20 \%{ }^{7}$. La prévalence du TSAF chez les enfants des Premières Nations vivant sur une réserve a diminué au fil du temps : elle était de 0,5\% selon l'Enquête régionale sur la santé des Premières Nations de 2015-2016, contre $1,8 \%$ en $2002-2003^{23}$.

L'écart entre le taux de prévalence des enfants et adolescents autochtones vivant hors réserve et celui des enfants et adolescents non autochtones ne peut pas s'expliquer uniquement par des différences sur le plan de l'exposition prénatale à l'alcool. Des facteurs interdépendants et complexes ont été définis et associés au TSAF et à l'exposition prénatale à l'alcool. Ces facteurs comprennent les effets dévastateurs de la colonisation, notamment les expériences vécues dans les pensionnats, et la marginalisation socioéconomique dont les peuples autochtones continuent d'être victimes ${ }^{24}$.

\section{Points forts et limites}

Un des points forts de cette étude est que l'ECSEJ de 2019 est une enquête nationale fondée sur la population qui couvre un large éventail de thèmes et permet d'examiner en profondeur les caractéristiques sociodémographiques et sanitaires des enfants et des adolescents canadiens. À l'heure actuelle, l'ECSEJ est la seule source de données qui permette d'établir des estimations de la prévalence du TSAF à l'échelle du pays. Cependant, bien qu'elle soit représentative de $98 \%$ de la population canadienne, l'ECSEJ ne tient pas compte des groupes de population constitués d'enfants et d'adolescents dont on sait qu'ils présentent une prévalence plus élevée de $\mathrm{TSAF}^{21,25}$, en l'occurrence ceux qui vivent dans une réserve des Premières Nations et d'autres lieux de vie autochtones des provinces, dans des foyers d'accueil et en institution. Ces exclusions ont sans doute entraîné une sous-estimation de la prévalence du TSAF chez les enfants et les adolescents canadiens.

Bien qu'il soit suffisant pour être présenté, le nombre de cas de TSAF ayant fait l'objet de notre analyse était petit. Nous n'avons pas pu présenter d'analyse du rôle des facteurs sociodémographiques chez les enfants et adolescents atteints du TSAF en raison de la variabilité d'échantillonnage élevée. Par ailleurs, comme le diagnostic de TSAF peut faire l'objet de stigmatisation, il pourrait avoir été sous-déclaré ${ }^{15}$.

D'après notre analyse, plus des trois quarts des enfants et adolescents atteints d'un TSAF avaient aussi un problème de santé concomitant. Popova et ses collaborateurs ${ }^{14}$ ont émis l'hypothèse que le nombre de problèmes de santé concomitants chez les personnes atteintes d'un TSAF pourrait expliquer pourquoi les estimations de prévalence du TSAF sont moins élevées que prévu : ces autres problèmes de santé pourraient faire l'objet de consultations médicales spécifiques, conduisant à un sous-diagnostic de leur cause sousjacente (à savoir un TSAF) ${ }^{14}$.

Les méthodes passives de collecte de données, comme l'utilisation de l'ECSEJ, sont les plus efficaces et les plus représentatives, mais elles tendent à produire les estimations les plus faibles, pour les raisons décrites ci-dessus. Les méthodes actives en milieu clinique donnent des estimations de la prévalence plus élevées et plus de détails sur les cas de TSAF, mais elles sont aussi plus dispendieuses et exigent plus de temps, c'est pourquoi elles ciblent généralement des zones géographiques ou des groupes de population qui présentent un intérêt particulier ${ }^{4}$.

\section{Conclusion}

La surveillance nationale du TSAF est nécessaire pour mieux comprendre la prévalence estimée et pour établir des valeurs de référence qui permettront d'évaluer l'efficacité des mesures de santé publique dans l'avenir. En utilisant les données de l'ECSEJ de 2019, nous avons estimé la prévalence du TSAF chez les enfants et adolescents canadiens vivant en ménage privé à 1 pour 1000 .

À notre connaissance, cette analyse est la première estimation nationale de la prévalence du TSAF au Canada. Elle constitue un point de départ à une meilleure connaissance du fardeau associé à ce problème de santé évitable. En raison des défis actuels, il pourrait être nécessaire de combiner des méthodes de surveillance et sources de données passives et actives pour réaliser la surveillance du TSAF au Canada.

\section{Remerciements}

Les données présentées dans les tableaux sont fondées sur les résultats de l'Enquête canadienne sur la santé des enfants et des jeunes (ECSEJ) menée par Statistique Canada en 2019. Nous souhaitons également remercier le Comité consultatif sur le bien-être des femmes autochtones et la $\mathrm{D}^{\text {re }}$ Jocelynn Cook pour leurs précieux commentaires sur le manuscrit.

\section{Conflits d'intérêts}

Aucun.

\section{Contributions des auteurs et avis}

SP a analysé les données et AP et SP ont rédigé l'article. Tous les auteurs ont participé à la conception de l'étude, à l'analyse des données, à l'interprétation des résultats et à la révision critique du manuscrit et ils en ont approuvé la version finale.

Le contenu de l'article et les points de vue qui y sont exprimés n'engagent que les auteurs; ils ne correspondent pas nécessairement à ceux du gouvernement du Canada.

\section{Références}

1. Cook JL, Green CR, Lilley CM, et al.; Canada Fetal Alcohol Spectrum Disorder Research Network. Fetal alcohol spectrum disorder: a guideline for diagnosis across the lifespan. CMAJ. 2016; 188(3):191-197. https://doi.org/10.1503 /cmaj.141593

2. Jones KL, Smith DW, Ulleland CN, Streissguth P. Pattern of malformation in offspring of chronic alcoholic mothers. Lancet. 1973;301(7815):1267-1271. https://doi.org/10.1016/S0140-6736(73) 91291-9

3. Harding K, Flannigan K, McFarlane A. Policy action paper: toward a standard definition of fetal alcohol spectrum disorder in Canada. Vancouver (BC) : CanFASD; 2019.

4. May PA, Gossage JP. Estimating the prevalence of fetal alcohol syndrome. A summary. Alcohol Res Health. 2001;25(3):159-167. 
5. Pei J, Reid-Westoby C, Siddiqua A, et al. Teacher-reported prevalence of FASD in kindergarten in Canada: association with child development and problems at home. J Autism Dev Disord. 2021;51(2):433-443. https:// doi.org/10.1007/s10803-020-04545-w

6. Fox DJ, Pettygrove S, Cunniff C, et al. Fetal alcohol syndrome among children aged 7-9 years - Arizona, Colorado, and New York, 2010. MMWR Morb Mortal Wkly Rep. 2015;64(3):54-57.

7. Fitzpatrick JP, Latimer J, Olson HC, et al. Prevalence and profile of neurodevelopment and fetal alcohol spectrum disorder (FASD) amongst Australian Aboriginal children living in remote communities. Res Dev Disabil. 2017; 65:114-126. https://doi.org/10.1016/j .ridd.2017.04.001

8. Flannigan K, Unsworth K, Harding K. The prevalence of fetal alcohol spectrum disorder. Vancouver (BC) : CanFASD; 2018.

9. Chudley AE. Diagnosis of fetal alcohol spectrum disorder: current practices and future considerations. Biochem Cell Biol. 2018;96(2):231236. https://doi.org/10.1139/bcb-2017 $-0106$

10. Statistique Canada. Enquête canadienne sur la santé des enfants et des jeunes (ECSEJ). Ottawa (Ont.) : Statistique Canada [dernière modification : 11 février 2019; consultation le 6 octobre 2020]. En ligne à : https://www23.statcan.gc.ca/imdb /p2SV_f.pl?Function = getSurvey $\&$ SDDS $=5233$

11. Statistique Canada. Enquête canadienne sur la santé des enfants et des jeunes (ECSEJ) de 2019 : guide de l'utilisateur. Ottawa (Ont.) : Statistique Canada; 2020.

12. Statistique Canada. Enquête canadienne sur la santé des enfants et des jeunes - 2019 : Questionnaire. Ottawa (Ont.) : Statistique Canada; 2019 [consultation le 6 octobre 2020]. En ligne à : https://www23.statcan.gc.ca /imdb/p3Instr_f.pl? Function = assembleInstr\&lang = en\&Item _Id $=1209093$
13. Statistical Analysis System Enterprise Guide version 7.1. Cary (NC) : SAS Institute Inc.

14. Popova S, Lange S, Shield K, et al. Comorbidity of fetal alcohol spectrum disorder: a systematic review and metaanalysis. Lancet. 2016;387(10022):978987. https://doi.org/10.1016/S0140 $-6736(15) 01345-8$

15. Popova S, Lange S, Poznyak V, et al. Population-based prevalence of fetal alcohol spectrum disorder in Canada. BMC Public Health. 2019;19(1):845. https://doi.org/10.1186/s12889-019 $-7213-3$

16. Thanh NX, Jonsson E, Salmon A, Sebastianski M. Incidence and prevalence of fetal alcohol spectrum disorder by sex and age group in Alberta, Canada. J Popul Ther Clin Pharmacol. 2014;21(3):e395-404.

17. Mutch RC, Watkins R, Bower C. Fetal alcohol spectrum disorders: notifications to the Western Australian Register of Developmental Anomalies. J Paediatr Child Health. 2015;51(4):433-436. https://doi.org/10.1111/jpc.12746

18. May PA, Baete A, Russo J, et al. Prevalence and characteristics of fetal alcohol spectrum disorders. Pediatrics. 2014;134(5):855-866. https://doi.org /10.1542/peds.2013-3319

19. May PA, Chambers CD, Kalberg WO, et al. Prevalence of fetal alcohol spectrum disorders in 4 US communities. JAMA. 2018;319(5):474-482. https:// doi.org/10.1001/jama.2017.21896

20. May PA, Gossage JP, Kalberg WO, et al. Prevalence and epidemiologic characteristics of FASD from various research methods with an emphasis on recent in-school studies. Dev Disabil Res Revs. 2009;15(3):176-192. https://doi.org/10.1002/ddrr.68

21. Popova S, Lange S, Probst C, Parunashvilia N, Rehmabde J. Prevalence of alcohol consumption during pregnancy and Fetal Alcohol Spectrum Disorders among the general and Aboriginal populations in Canada and the United States. Eur J Med Genet. 2017;60(1):32-48. https://doi .org/10.1016/j.ejmg.2016.09.010
22. Montag AC, Romero R, Jensen $\mathrm{T}$, et al. The prevalence of fetal alcohol spectrum disorders in an American Indian community. Int J Environ Res Public Health. 2019;16(12):2179. https:// doi.org/10.3390/ijerph16122179

23. First Nations Information Governance Centre. FNIGC data online: percentage of First Nations children diagnosed with a mental health condition or FASD [database]. Akwesasne (Ont.) : FNIGC [consulté le 2 juin 2021]. En ligne à : https://fnigc.ca/dataonline/ charts-list?term_node_tid_depth _1 = All\&term_node_tid_depth $=10$ \&keys $=$ FASD

24. Tait C. Syndrome d'alcoolisation fœtale chez les peuples autochtones du Canada : examen et analyse des répercussions intergénérationnelles liées au régime des pensionnats. Ottawa (Ont.) : Fondation autochtone de guérison; 2003.

25. Bower C, Watkins RE, Mutch RC, et al. Fetal alcohol spectrum disorder and youth justice: a prevalence study among young people sentenced to detention in Western Australia. BMJ Open. 2018;8(2):e019605. https://doi .org/10.1136/bmjopen-2017-019605 